\title{
Post-Training Lesions of the Medial Prefrontal Cortex Interfere with Subsequent Performance of Trace Eyeblink Conditioning
}

\author{
Barbara Simon, ${ }^{1,2}$ Bryan Knuckley, ${ }^{1}$ John Churchwell, ${ }^{1}$ and Donald A. Powell ${ }^{1,2,3}$ \\ ${ }^{1}$ Shirley L. Buchanan Neuroscience Laboratory, Dorn Veterans Affairs Medical Center, Columbia, South Carolina 29209-1639, 2Department of Psychology, \\ University of South Carolina, Columbia, South Carolina 29208, and ${ }^{3}$ Department of Neuropsychiatry and Behavioral Science, University of South Carolina \\ School of Medicine, Columbia, South Carolina 29208
}

\begin{abstract}
Rabbits were trained on trace eyeblink (EB) conditioning until they reached a criterion of 10 consecutive EB conditioned responses (CRs). Electrolytic lesions were made in the medial prefrontal cortex (mPFC) centered on the prelimbic area (Brodmann's area 32), at five different intervals after training. These included immediately, $24 \mathrm{~h}, 1$ and 2 weeks, and 1 month after training. Separate groups of animals received sham lesions at these same intervals after training. After a 2 week postoperative recovery period, all animals were retested for $3 \mathrm{~d}$ on trace conditioning, using the same parameters used during preoperative training. Mean EB conditioning performance deficits occurred in the animals with mPFC lesions compared with sham-lesioned animals on the first day of retesting in all five groups. However, by the second or third day of retesting, the rabbits with lesions were performing at a level that was comparable with that of sham animals. Rabbits that received more posterolateral lesions of the neocortex did not, however, show postoperative conditioning deficits. A comparison of percentage EB CRs of animals with postoperative training with that of animals that received mPFC lesions before training suggests that the $\mathrm{mPFC}$ post-training lesions produce damage to a retrieval process and not to a storage site or an acquisition process.
\end{abstract}

Key words: rabbits; cingulate cortex; prelimbic cortex; classical conditioning; memory; recall

\section{Introduction}

The prefrontal cortex (PFC) has been related to a number of conditions, suggesting that this structure participates in learning and memory processes (Goldman-Rakic, 1995). Recent studies have investigated the role of the PFC in classical (pavlovian) conditioning. For example, several studies have demonstrated that the medial PFC (mPFC) is intimately involved in the acquisition of pavlovian-conditioned autonomic responses (Buchanan and Powell, 1982; Buchanan et al., 1985; Frysztak and Neafsey, 1991, 1994; Powell et al., 1994), but other studies have shown that mPFC damage has no effect on simple somatomotor eyeblink (EB) classical conditioning (Buchanan and Powell, 1982; Weible et al., 2000; McLaughlin et al., 2002; Powell et al., 2005). However, it has also been reported that, under conditions that are less than optimal for learning to occur, such as during trace conditioning or partial reinforcement, $\mathrm{mPFC}$ lesions also impair EB conditioning (Kronforst-Collins and Disterhoft, 1998; Weible et al., 2000; McLaughlin et al. 2002; Powell et al., 2005). During

Received July 20, 2005; revised Sept. 7, 2005; accepted 0ct. 4, 2005.

This work was supported by Department of Veterans Affairs medical research funds, awarded to the Veterans Affairs Medical Center (Columbia, SC). We thank Elizabeth Hamel for manuscript preparation and Andrew Pringle Jr for assistance with preparation of the illustrations.

Correspondence should be addressed to Dr. Donald A. Powell, Shirley L. Buchanan Neuroscience Laboratory, 151A, William Jennings Bryan Dorn Veterans Affairs Medical Center, 6439 Garners Ferry Road, Columbia, SC 292091639. E-mail: donnie.powell@med.va.gov.

DOI:10.1523/JNEUROSCI.3003-05.2005

Copyright $\odot 2005$ Society for Neuroscience $\quad$ 0270-6474/05/2510740-07\$15.00/0 trace conditioning, a temporal gap occurs between the termination of the conditioned stimulus (CS) and the onset of the unconditioned stimulus (US), which can be contrasted with delay conditioning, in which the CS overlaps the US and the two stimuli coterminate. Lesions of the mPFC have no effect on EB delay conditioning but impair trace EB conditioning (KronforstCollins and Disterhoft, 1998; Weible et al., 2000; McLaughlin et al., 2002). These findings, taken as a whole, suggest the generalization that, under conditions that are optimal for acquisition for somatomotor learning (e.g., simple delay conditioning), mPFC processing plays a minimal role in somatomotor pavlovian conditioning, but under conditions in which learning occurs more slowly, mPFC processing may be important for acquisition to occur. These considerations led us to question whether these interesting effects are attributable to acquisition or storage and/or retrieval of CS information. This question motivated us to perform a set of experiments in which, rather than producing lesions before training, as in our previous experiments, the rabbits were first trained and then lesions were made in the $\mathrm{mPFC}$ (primarily centered on area 32), with the idea that processing by area 32 is necessary for the optimal processing of information critical to somatomotor behaviors (Powell et al., 2001). The results of this study showed that, although delay conditioning was significantly impaired by post-training $\mathrm{mPFC}$ lesions, a much more profound decrement in performance was obtained when trace conditioning was used. In these experiments, the lesion was made shortly after training was completed. Thus, it was impossible to conclude un- 
equivocally that damage to the $\mathrm{MPFC}$ produces a long-term effect on performance of the EB conditioned response (CR). An alternative hypothesis is that $\mathrm{mPFC}$ lesions interrupted a timedependent consolidation process that is necessary for performance of the learned response. In the present study, we followed up these findings by performing post-training mPFC lesions at several points after training, including immediately (within $6 \mathrm{~h}$ ), $24 \mathrm{~h}, 1$ and 2 weeks, and 1 month after training.

\section{General Methods}

Animals. The animals used were 99 male and female New Zealand Albino rabbits, obtained from a supplier (Robinson's Services, Winston-Salem, NC) licensed by the United States Department of Agriculture. Eight animals either died or were killed because of illness before being tested. All animals were maintained in an animal facility accredited by the American Association for Assessment and Accreditation of Animal Laboratory Care International. A $12 \mathrm{~h}$ light/dark cycle was used, with lights on at 7:00 A.M. All behavioral testing was conducted during the daylight portion of the light/dark cycle. Food and water were available ad libitum. All United States Public Health Service and Department of Veterans Affairs regulations regarding animal welfare were followed.

Surgical procedures. All surgery was performed under aseptic conditions. Animals were anesthetized with ketamine hydrochloride $(55 \mathrm{mg} /$ $\mathrm{kg}$, i.m.) supplemented by acepromazine malate $(2.2 \mathrm{mg} / \mathrm{kg}$, i.m. $)$ and xylazine $(3.0 \mathrm{mg} / \mathrm{kg}, \mathrm{i} . \mathrm{m}$. $)$. The anesthetized animals were placed in a David Kopf Instruments (Tujunga, CA) stereotaxic frame with bregma $1.5 \mathrm{~mm}$ higher than lambda. After retracting the overlying tissue, holes were drilled in the skull over appropriate areas of the mPFC with a dental drill. Stainless-steel electrodes (Frederick Haer, Brunswick, ME) were then stereotaxically lowered into the areas of interest, and electrolytic lesions were made using a Grass DC lesion maker (AstroMed, West Warwick, RI) ( $3 \mathrm{~mA}$ for $20 \mathrm{~s}$ ). The coordinates, with reference to bregma, the midline, and dura, were: anterior $(\mathrm{A}), 0$; lateral $(\mathrm{L}), \pm 0.9$; and ventral (V), 1.5 and $3.0 ; \mathrm{A}, 2 ; \mathrm{L}, \pm 0.9$, and $\mathrm{V}, 1.5$ and $3.0 ; \mathrm{A}, 4 ; \mathrm{L}, \pm 0.9$; and $\mathrm{V}, 1.5$ and $3.0 ; \mathrm{A}, 6$; $\mathrm{L}, \pm 0.9$; and $\mathrm{V}, 1.3,3.5$, and $5.5 ; \mathrm{A}, 8 ; \mathrm{L}, \pm 0.9$; and $\mathrm{V}, 2.0$, 4.0, 5.0, and 6.0; and A, 10.0; L, \pm 0.9 ; and V, 2.0, 4.0, and 5.0. Sham control animals were anesthetized, and holes were drilled in the skull, as for the lesioned subjects, but no further brain manipulations took place. For all animals, a 2 week interval separated surgery and retesting.

Apparatus and procedure. Experimental contingencies were controlled by a personal computer-based data acquisition system (MACRO, Columbia, SC), supplemented by solid-state transistor-transistor logic programming devices. The EB responses were recorded on a Grass model 7 polygraph (AstroMed) equipped with EMG preamplifiers. During conditioning, the output of the polygraph was connected to the computer where analog-to-digital (A-D) conversion was performed in real time. The shock US was delivered by a Grass model S88 stimulator (AstroMed) equipped with constant-current and stimulus isolation units. During the experiment, animals were restrained in Plexiglas rabbit restrainers (Gormezano, 1966) in ventilated, sound- and light-attenuating commercial animal enclosures (Industrial Acoustics, Bronx, NY). The CS was a 500 ms, $1200 \mathrm{~Hz}, 75 \mathrm{~dB}$ tone. A $100 \mathrm{~ms}, 3 \mathrm{~mA} \mathrm{AC}(200 \mathrm{~Hz})$ electric shock train was the US. This US was delivered periorbitally through previously implanted stainless-steel wound clips. Each session consisted of 100 trials with a $30 \pm 5 \mathrm{~s}$ intertrial interval. The EB response was measured via electrodes consisting of Tru-Chrome dental wire acutely inserted under the eyelids before the beginning of each session. Insertion of these electrodes caused neither apparent discomfort nor any signs of infection or irritation. Leads for the EB electrodes were connected to a Grass model $7 \mathrm{P} 3$ preamplifier and integrator set in its integrator mode. The preamplifier was calibrated so that a $0.10 \mathrm{~V}$ change across the electrodes corresponded a $0.5 \mathrm{~mm}$ movement of the eyelids. During conditioning, the EB response was recorded by the A-D converter of the computer, which sampled at $1000 \mathrm{~Hz}$ beginning $50 \mathrm{~ms}$ before tone onset and continuing until US offset. Each animal received two initial habituation sessions in which they were loosely restrained in the chamber, but no stimuli were presented. These two sessions were followed by daily sessions of trace conditioning in which the CS was presented paired with the US but with
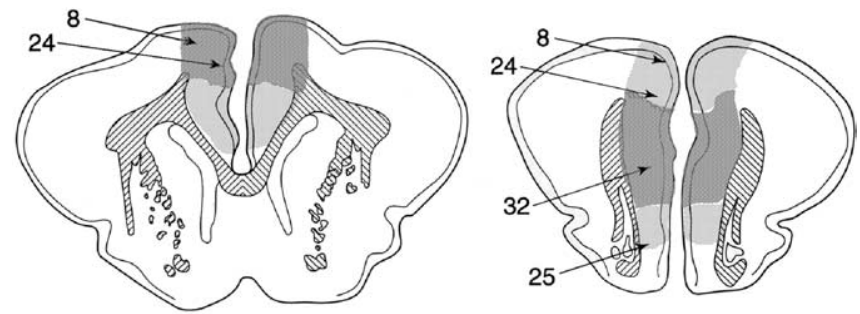

A.5
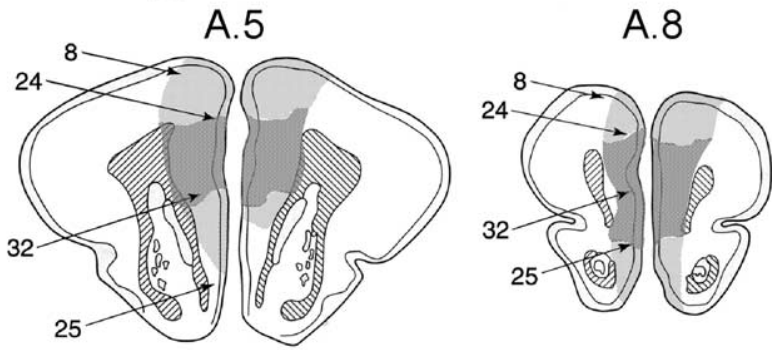

A. 6

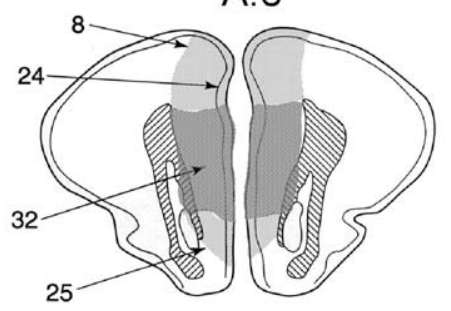

A. 7

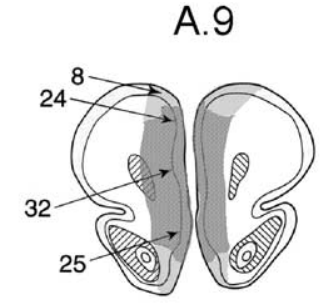

A. 10

Figure 1. Diagrams of frontal sections of the rabbits' brains, showing maximal (light stippling) and minimal (dark stippling) damage to the $\mathrm{MPFC}$ for rabbits in the present experiments. Approximate stereotaxic coordinates anterior (A) to bregma are indicated. Lower limits for the Brodmann's areas are also indicated as follows: 8 , prefrontal eye fields; 24 , anterior cingulate cortex; 32, prelimbic area; and 25, infralimbic area.

a $500 \mathrm{~ms}$ interval separating CS termination and US onset. Training continued until the animal executed 10 consecutive EB CRs. Surgery occurred at varying times after this criterion was met. In the main experiment, five intervals included 6 and $24 \mathrm{~h}$ and 1,2, and 4 weeks after training to the criterion. All animals were allowed a 2 week postsurgical recovery period, at which time they were retested on the trace conditioning task for 3 consecutive days, using the same parameters that were used during original acquisition.

Histology. After behavioral testing was completed, the animals were killed with pentobarbital and perfused transcardially with physiological saline and $10 \%$ formalin. The brain was then removed; it was quick frozen in 2-methylbutane and then sliced in the coronal plane in a cryostat. Forty micrometer sections throughout the lesions were stained with thionin. The lesions were then located microscopically, and line drawings were made of the appropriate sections using a Leitz (Wetzlar, Germany) drawing tube and microscope. The extent of the lesion was then determined by superimposing these drawings onto plates from the atlas by Shek et al. (1986).

Data reduction and analysis. The criterion for an EB CR was a $0.10 \mathrm{~V}$ change from baseline during CS presentation or the trace period, with a latency of $>100 \mathrm{~ms}$. CR latency was recorded as the time from CS onset until the EB response first exceeded the criterion. EB CRs with a latency of $<100 \mathrm{~ms}$ were considered $\alpha$ responses (i.e., reflex responses to the tone) and were discarded (Kimble, 1961). This occurred on $<1 \%$ of the trials, however. CR amplitude was recorded as the maximal amplitude change (in millivolts) from baseline during the CS-US period.

\section{Results}

Histology

Figure 1 shows diagrams through the MPFC of the rabbit's brain showing the maximal (light stippling) and minimal (dark stip- 
Table 1. Mean trials to criterion \pm 1 SEM during preoperative training

\begin{tabular}{lll}
\hline Interval & Sham & Lesion \\
\hline $6 \mathrm{~h}$ & $283.1 \pm 17.0$ & $265.9 \pm 16.3$ \\
$24 \mathrm{~h}$ & $270.7 \pm 18.8$ & $319.8 \pm 25.2$ \\
1 week & $273.8 \pm 18.8$ & $293.8 \pm 18.8$ \\
2 weeks & $293.0 \pm 18.8$ & $273.0 \pm 18.8$ \\
4 weeks & $251.5 \pm 14.5$ & $316.6 \pm 17.0$ \\
\hline
\end{tabular}

pling) damage to the mPFC. The Brodmann's areas are also indicated. Figure 1 shows that maximal damage included all of areas 32 and 24, extended dorsally into the shoulder portion of the midline prefrontal region, and included in many cases portions of the prefrontal eye fields (area 8). Damage also extended ventrally into area 25 in several rabbits in each of the groups with $\mathrm{mPFC}$ lesions. However, as can be seen in Figure 1, the damage common to all lesions was centered on area 32.

\section{Preoperative performance}

Table 1 shows the mean trials to reach a criterion of 10 consecutive eyeblink CRs for each group of animals. ANOVA of the trials to criterion data revealed no significant differences between the five groups $\left(F_{(4,78)}=1.36 ; p=0.255\right)$. Moreover, a correlation analysis was conducted on the trials to criterion scores and percentage EB CRs on the first retest day when the lesion-induced deficit was greatest (see below, Postoperative performance). This analysis yielded a nonsignificant correlation coefficient $(r=$ 0.126 ; df $=78$; $p>0.05$ ).

\section{Postoperative performance}

Figure $2 A-E$ shows mean percentage \pm 1 SEM EB CRs over each of the 3 retest days for each of the groups. As can be seen, each of the five postoperative intervals resulted in deficits in EB responding in the $\mathrm{mPFC}$ lesion groups compared with the sham-lesioned animals, although this performance deficit became smaller over days, until by the end of testing on day 3 , the sham and lesion groups were comparable at all five testing intervals. Thus, there was a recovery of function with additional postoperative training.

ANOVA was performed on these data using the five postoperative intervals (five levels) and sham versus lesion (two levels) as nonrepeated factors. The three daily reacquisition sessions served as a repeated factor (three levels). The results of this ANOVA revealed a significant interval-group effect $\left(F_{(4,79)}=\right.$ $4.64 ; p<0002)$, a significant lesion versus sham effect $\left(F_{(1,79)}=\right.$
$18.4 ; p<00001)$, and a significant session effect $\left(F_{(2,158)}=41.35\right.$; $p<00001)$. The sham/lesion-by-session $\left(F_{(2,158)}=22.5 ; p<\right.$ $00001)$ and interval-group-by-session $\left(F_{(10,158)}=3.45 ; p<\right.$ $00015)$ interactions were also significant. No other effects or interactions were significant.

Post hoc tests using the Newman-Keuls procedure were used to examine the source of the significant interval-group effect and the interval-group-by-session interaction. These tests revealed that the performance of the 1 week group was significantly lower than each of the other four groups (largest $p<0.05$ ), which, however, did not differ significantly from each other. The lower scores of this group on sessions 1 and 2, but not session 3, also accounted for the group-by-session effect. On both days 1 and 2, the 1 week group differed from all the other four groups (largest $p<0.05)$, but these groups did not differ significantly from each other. However, there were no significant effects on session 3.

The sham/lesion-by-session interaction was further analyzed by separate planned ANOVAs of each of the five interval groups. These analyses revealed a lesion effect on day 1 for all five groups (smallest $F_{(1,14)}=5.6 ; p<0.03$, for the 24 h group). In addition, the lesion versus sham effect was significant for the 1 month group for day $2\left(F_{(1,12)}=4.93 ; p<0.04\right)$. However, the lesion versus sham effect was not significant for any other group on days 2 or 3.

\section{CR and UR latencies and amplitudes}

Two-way ANOVA, including lesion (two levels) and intervalgroup (five levels) as factors, was conducted on CR and UR amplitude and latency. Significant interval-group $\left(F_{(4,59)}=2.95\right.$; $p<0.03)$ and lesion $\left(F_{(1,59)}=4.49 ; p<0.04\right)$ effects were obtained for CR amplitude, but CR latency and UR latency and amplitude were not statistically significant (smallest $F_{(1,59)}=$ $2.14 ; p>0.0892$ ). The significant interval-group and lesion effects for CR amplitude were further analyzed by means of the Newman-Keuls post hoc procedure. This analysis revealed that CR amplitude was significantly lower in the 1 week and $24 \mathrm{~h}$ groups (amplitude, 214 and $191 \mathrm{mV}$, respectively) than in the other three groups $(381,387$, and $349 \mathrm{mV})$. Separate one-way ANOVAs of the differences in CR amplitude between the lesion and sham groups on day 1 for each of the five groups revealed a significant $F$ value only for the 1 week group $\left(F_{(1,14)}=6.5 ; p<\right.$ $0.03)$. All other sham/lesion differences were nonsignficant (largest $p<0.09$ ).
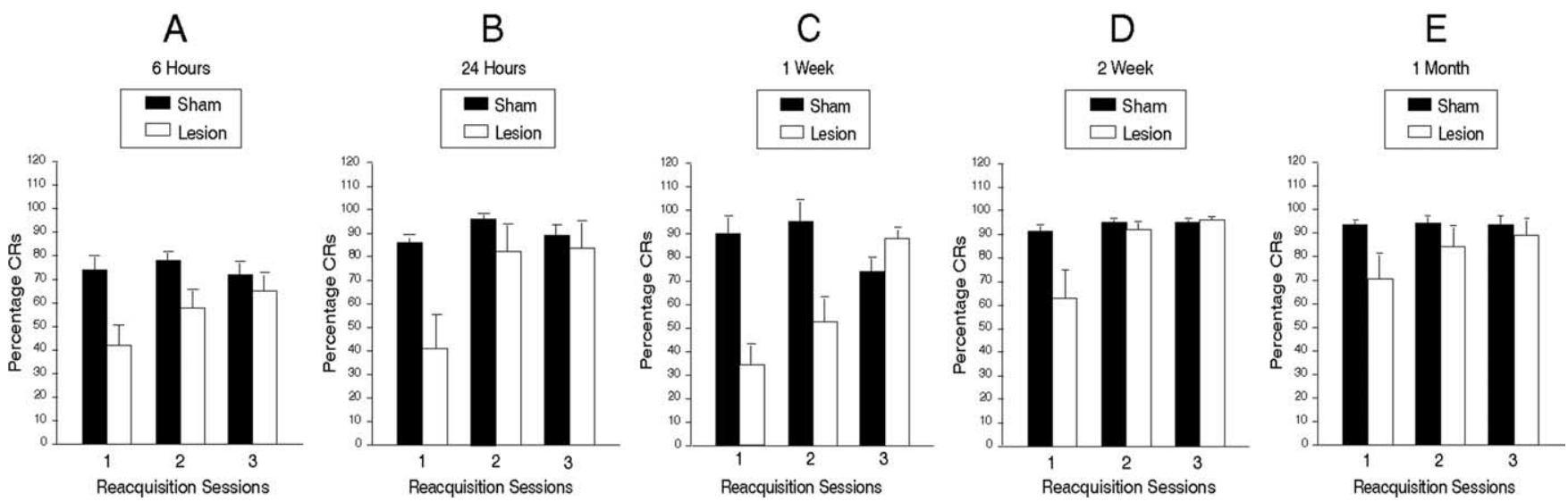

Figure 2. Mean percentage EB CRs $\pm 1 \mathrm{SEM}$ in post-training sham and $\mathrm{mPFC}$ lesion groups as a function of the three postoperative test sessions. Lesions were made at five different intervals after original acquisition to criterion as indicated: $\boldsymbol{A}, 6 \mathrm{~h} ; \boldsymbol{B}, 24 \mathrm{~h} ; \boldsymbol{C}$, 1 week; $\boldsymbol{D}, 2$ weeks; and $\boldsymbol{E}, 1$ month. 

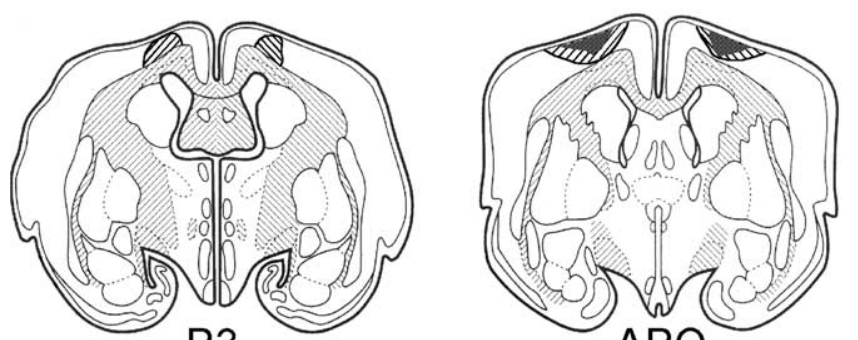

P3

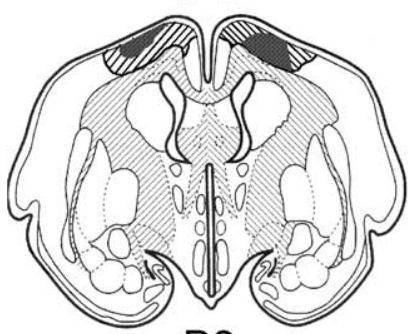

$\mathrm{P} 2$

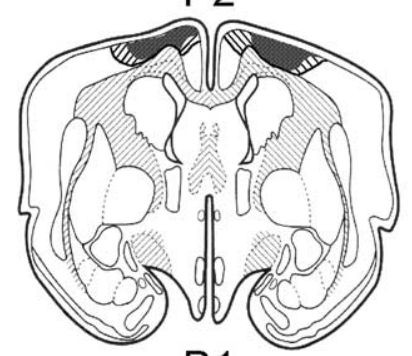

$\mathrm{P} 1$

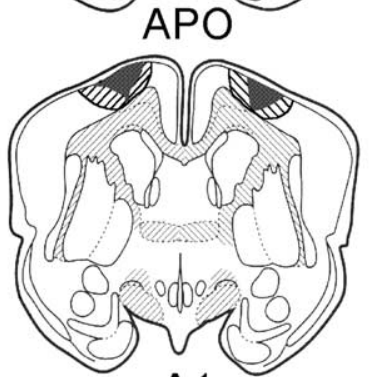

A1

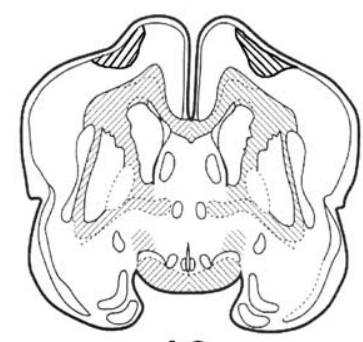

A2

Figure 3. Diagrams of frontal sections through the rabbits' brains, showing maximal (striped) and minimal (stippled) damage to the posteriolateral cortex for animals that participated in experiment 2. Approximate stereotaxic coordinates anterior $(A)$ and posterior $(P)$ to bregma are also indicated. APO, Bregma.

\section{Discussion}

We conclude from these experiments that post-training lesions of the $\mathrm{mPFC}$ produces a relatively permanent deficit in trace eyeblink conditioning in the rabbit. In a previous study (see above), which investigated the effects of post-training mPFC damage on trace EB conditioning performance, only a single interval was used, namely, immediately after training (Powell et al., 2001). It was thus impossible to determine from these results whether the impairment was permanent or whether the lesion interfered with a time-dependent consolidation process. The present data show conclusively that this deficit is relatively permanent and would appear to suggest that the deficit produced in trace eyeblink conditioning in animals with prefrontal damage is attributable to a retrieval or storage problem and not an interference with acquisition. Moreover, the long-lasting effects of the lesions suggest that it does not interfere with a time-dependent consolidation process.

There was, however, some indication of a possible consolidation process during the first week after training because both the $24 \mathrm{~h}$ and 1 week groups had lower EB amplitude CRs than the other groups during the first day of retesting, and the CR amplitude of the lesion group was lower than the that of the sham group during day 1 for the 1 week group. Moreover, percentage CRs were lower for the 1 week group compared with the other four groups for retest days 1 and 2. We have no explanation for these anomalous effects because there are no consolidation processes, of which we are aware, that are known to be ongoing

during this period that would not have been evident earlier in the 6 and 24 h groups.

However, several other aspects of these data raise further questions. First, an obvious question is whether damage specifically to the $\mathrm{mPFC}$ is critical for these mnemonic deficits or whether damage to other midline cortical structures would produce the same effect. Thus, in a second experiment, lesions in the posteriolateral neocortex were made on animals 1 week after their meeting the training criterion. A second question concerns the relative impairment produced in the present experiment compared with the deficits normally shown in prefrontal lesioned animals when the lesion is made preoperatively. A third experiment thus compared the group with 1 week post-training lesions with animals that received preoperative lesions.

\section{Experiment 2 Methods}

In the second experiment, animals were trained to a criterion, as described above, and 1 week later, more posterolateral lesions were made similar in extent to those made in experiment 1 in the mPFC. Three lesions were made on each side of the brain. The coordinates used in this surgery were as follows: dorsoventral, $2.5 ; \mathrm{L}, 2.0$; and anteroposterior and posterior, 1.0 and 2.0. Preoperative as well as postoperative training was identical to that of experiment 1.

\section{Results \\ Histology}

Figure 3 shows diagrams of frontal sections of the rabbit's brain through the area of the lesion in the posterolateral neocortex. Striped represents maximal damage, whereas stippled represents minimal damage. As can be seen, damage occurred in the prefrontal cortex beginning just posterior to the anterior cingulate cortex that was damaged in experiment 1 . The extent of the damage in the posterolateral neocortical area appears to be comparable with that of the prefrontal area of experiment 1 .

\section{Postoperative performance}

Figure 4 shows eyeblink percentage CRs over $3 \mathrm{~d}$ of retraining in rabbits with lesions of the MPFC (from Fig. 2) and a second group of animals with posterior prefrontal lesions compared with sham surgeries 1 week after initial acquisition. Figure 4 shows that animals with posterior lesions were comparable with sham animals with mPFC lesions. It can also be seen in Figure 4 that animals with $\mathrm{mPFC}$ lesions were considerably impaired relative to both sham $\mathrm{mPFC}$ and posterior cortex lesions. ANOVA suggested that these differences were reliable. A significant sham versus lesion effect was obtained for test days $1\left(F_{(2,20)}=5.41 ; p<0.01\right)$ and 2 $\left(F_{(2,20)}=4.11 ; p<0.03\right)$. No significant lesion effect was obtained for day 3; however, Newman-Keuls post hoc tests showed that the mPFC-lesioned group was significantly different from both the sham $\mathrm{mPFC}$ and posterior cortical groups for the first $2 \mathrm{~d}$ of training $(p<0.05)$, but the latter two groups were not significantly different for any of the $3 \mathrm{~d}$.

\section{Discussion}

We conclude from the present data that the impairments in EB conditioning performance obtained in experiment 1 , as a result of post-training lesions to the $\mathrm{mPFC}$, were indeed attributable specifically to damage to areas 32 and 24, as shown in Figure 1. More posterior damage resulted in performance that was in all ways comparable with that of the sham animals in experiment 1 .

\section{Experiment 3 Methods}

Observation of the results of experiment 1 suggest that learning was not completely abolished by the lesion at any of the five 


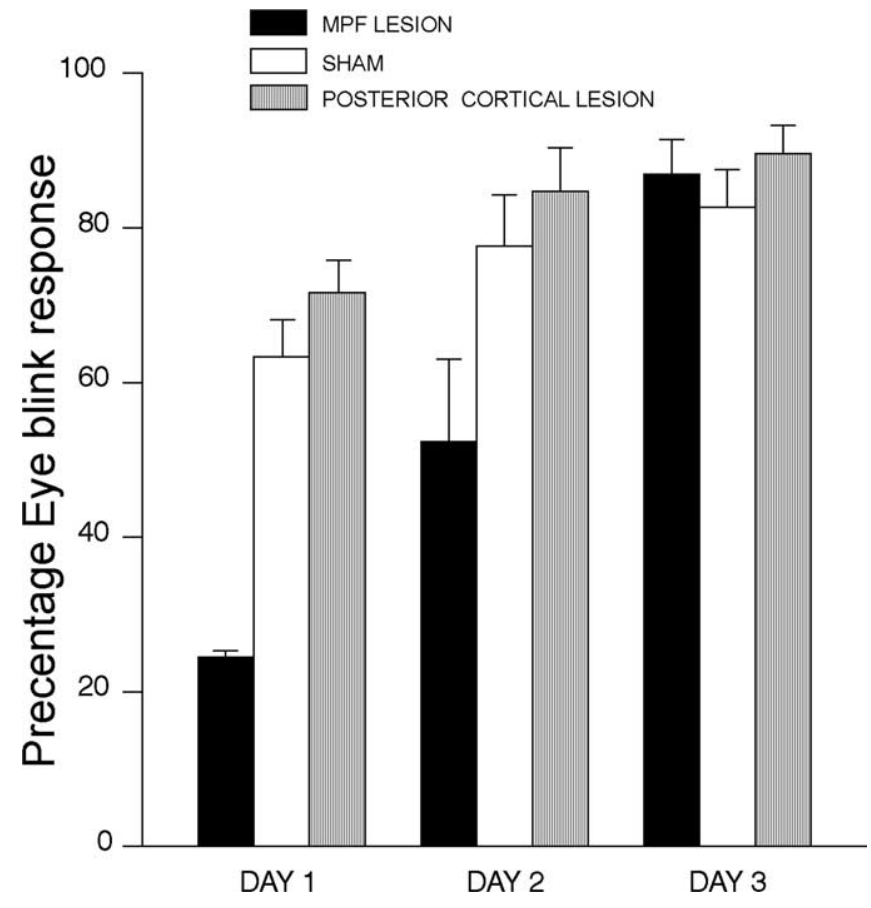

Figure 4. EB percentage CRs over $3 \mathrm{~d}$ of retraining in rabbits with lesions to the $\mathrm{mPFC}$ (MPF) or posterior prefrontal cortex or sham lesions 1 week after initial acquisition.

postoperative intervals. It could thus be the case that, rather than starting at a zero baseline, the performance of animals with mPFC damage is more similar to naive unoperated animals. That is, mPFC damage may interfere with memory for the task by destroying $\mathrm{mPFC}$ cells necessary for retrieval but may have no effect on acquisition mechanisms. To test this hypothesis, the animals with 1 week post-training lesions were compared with animals that received pretraining lesions during the first three sessions of acquisition. The acquisition and postoperative retest procedures were identical in this experiment to those of the previous two experiments.

\section{Results and Discussion}

The data from this experiment are shown in Figure 5, which shows eyeblink percentage CRs in rabbits with lesions made before training (pretraining lesions) and rabbits with lesions made 1 week after training (post-training lesions in the 1 week group from experiment 1 ) as a function of 3 acquisition or reacquisition days. As can be seen in Figure 5, the response of the preoperatively trained sham animals is very similar to that during retest day 1 of animals that received post-training mPFC lesions. ANOVA that compared the prelesioned and postlesioned animals' performance revealed a difference between the groups with preoperative versus postoperative surgery $\left(F_{(1,30)}=7.94 ; p<0.008\right)$. This difference was due to overall lower percentage CRs in the pretraining lesion group compared with the post-training lesion group. Indeed, both lesioned groups differed during days 1 and 2 from the sham-operated animals (smallest $F_{(1,30)}=5.92 ; p<$ 0.02 ). However, post hoc analysis using the Newman-Keuls procedure revealed that the lesion-post-training group did not differ from the sham-prelesion group on any of the $3 \mathrm{~d}(p>0.05)$.

However, the percentage CRs of the 1 week group differed from those of the other four groups, as described above. To further examine whether postoperative training produces animals that are naive with respect to conditioning, ANOVA compared

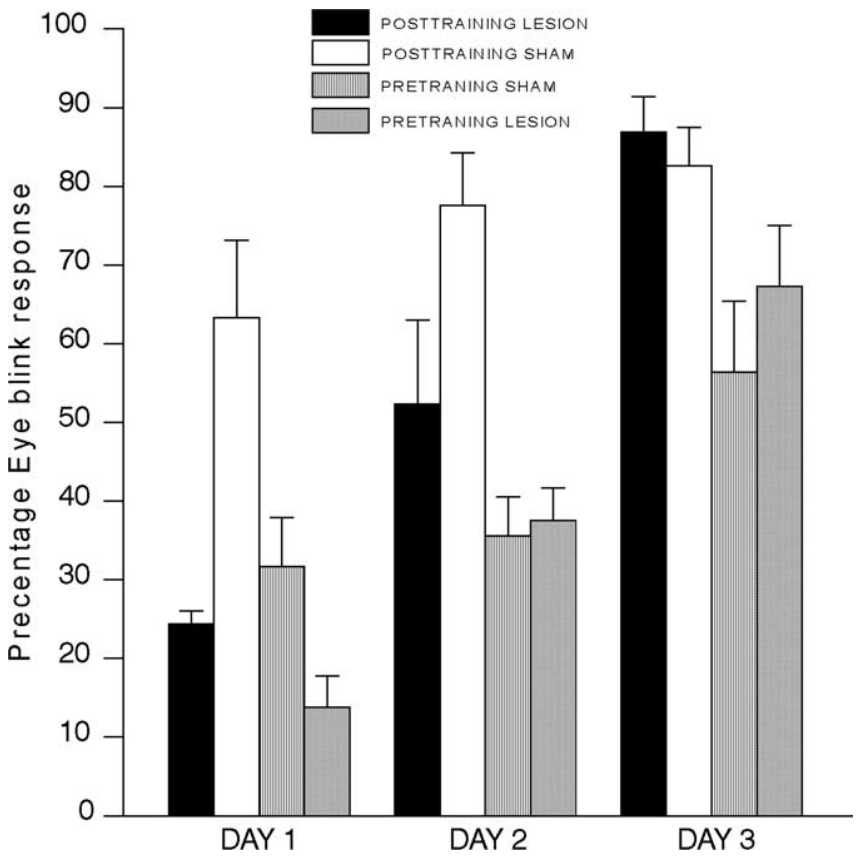

Figure 5. EB percentage CRs in rabbits with lesions before training (PRETRAINING) and rabbits with lesions made 1 week after training (POSTTRAINING) as a function of three acquisition or reacquisition sessions.

the sham pretraining data on day 1 with the data for each of the other four post-training group on retest day 1 . The retest percentage CRs of each of these four groups were significantly greater than that of the pretraining sham animals (smallest $p<0.05$ ). Thus, although retest percentage CRs of animals with postoperative training is not identical to percentage CRs on acquisition day 1 of naive animals, they are similar in that all post-training groups showed lower responding than trained animals, and all showed an acquisition function over the $3 \mathrm{~d}$ of retesting. The fact that animals with lesions begin acquisition at a higher level than acquisition of naive animals suggests that, whereas lesions to area 32 of the mPFC disrupt retrieval mechanisms, other structures may be involved in storage and working memory, and these structures are presumably intact and could therefore mediate the greater responding of the post-training lesioned animals during day 1.

We thus conclude from this experiment that postoperative damage to the mPFC does not impair the ability to learn the EB CR but indeed seems to impair a process related to retrieval. This conclusion is supported by the results of experiment 1 , in which all post-testing interval groups showed increases in performance over the 3 retest days.

\section{General Discussion}

The present data provide unequivocal evidence that the medial prefrontal cortex (i.e., areas 24 and 32) plays a role in memory for the trace eyeblink conditioned response. As noted above, in a previous study, we found that lesions of the MPFC immediately after training produced deficits in a recently learned EB conditioning task using both the delay and trace conditioning paradigms (Powell et al., 2001). In this study, the deficits produced in the trace conditioning task were considerably greater than those produced in the delay conditioning task (Powell et al., 2001). The present data confirm that these deficits are relatively permanent and thus that the mPFC is not only involved in mnemonic processing of a recently acquired trace conditioning task, but of a task 
which is more remotely removed from original training. As noted above, however, the significant poorer performance of the 1 week lesion group suggests the possibility that a consolidation process takes place between $1 \mathrm{~d}$ and 1 week after training. Clearly, this possibility deserves further study. Nevertheless, in general, the results of the present experiment cannot be interpreted as interference with a time-limited consolidation process.

It is of special note that a control experiment (experiment 2), which destroyed neocortical tissue more posterior to the mPFC, had no effect on subsequent performance of the trace EB CR. Thus, the results reported in experiment 1 could not be attributable to nonspecific damage of neocortical tissue. It thus seems quite likely that the effects produced are attributable to damage specific to area 32.

The data of the present experiments are compatible with a recent study that investigated trace eyeblink conditioning in rats (Takehara et al., 2003). In that experiment, rats received aspiration lesions of the hippocampus, prefrontal cortex, or cerebellum after they had learned a trace EB conditioning task to the criterion. These investigators found that hippocampal lesions impaired performance of the response only early during postoperative training; there was no lesion effect 2 weeks or 1 month after training. However, lesions of the $\mathrm{mPFC}$ produced postoperative performance deficits at 1 and 2 weeks and 1 month after training. Unlike the present study, however, the mPFC lesions in rats in this study appeared to produce increasing performance deficits as a function of the length of time after training that the lesion was made. As might be expected, based on the tremendous amount of information on the cerebellar control of eyeblink conditioning (for review, see Thompson et al., 2000), cerebellar lesions had a long-lasting effect at all three testing intervals. It is significant that, like the present study, in the study by Takehara et al. (2003), the mPFC lesions produced their greatest performance deficits on the first postoperative testing day, but their performance approached that of the sham animals by day 3 . It is difficult to understand why lesions that interfered with more remote memories had the greatest effect in this study, however. In fact, in the present study, the 1 month lesioned animals appeared to perform at a higher (but not significant) level than animals that received lesions earlier after training.

It is notable that post-training lesions in our previous study (Powell et al., 2001) had no effect on the accompanying heart rate conditioned response. As has been reported in several previous papers (Buchanan and Powell, 1982; Powell et al., 1994), pretraining lesions have deleterious effects on the conditioned heart rate decelerations usually obtained during classical EB conditioning. However, post-training lesions in our previous study had no such effect, suggesting that the effects of the lesions on the HR CR are attributable to interference during acquisition as opposed to performance of the response. Thus, the effects of area 32 lesions on the trace EB CR appear to be independent of the simultaneously occurring conditioned autonomic changes.

It has been suggested that the mPFC, especially the ventromedial area, is involved in the integration of emotional processes with other somatic and sensory information so that appropriate planning and adaptation to environmental circumstances can occur. One of the especially important aspects of this model of mPFC function is that somatomotor response selection mechanisms are guided by information integrated by the mPFC. Devinsky et al. (1995) as well as others (Fuster, 1997) have specifically suggested that a response selection mechanism is part of the function of the mPFC, although the focus of the former was on the anterior cingulate cortex, as opposed to the prelimbic region studied in the present experiments. The present findings suggest that mechanisms in the prelimbic area participate in such a response selection mechanism by providing a memory substrate for somatomotor responses that are appropriate for a specific environmental situation. Intrinsic connections between areas 32, 24, and 25 are known to be numerous (Buchanan et al., 1994). Thus, the processing of information by the prelimbic cortex may be integral to a somatomotor response selection mechanism residing in the anterior cingulate cortex. However, the fact that posttraining damage specifically to area 24 in our previous study (Powell et al., 2002) had no effect on the performance of the EB CR argues against such an interpretation.

It is especially notable that these effects are more pronounced when the trace paradigm is used. It has been shown previously by Kronforst-Collins and Disterhoft (1998), Weible et al. (2000), and McLaughlin et al. (2002) that pretraining mPFC lesions have a more dramatic effect on trace than delay conditioning. $\mathrm{mPFC}$ lesions also dramatically affect reversal conditioning but have no effect on initial differential discrimination of the EB CR (Chachich and Powell, 1999). Hippocampal lesions have a similar effect. Lesions of the hippocampus have little effect on delay EB conditioning but dramatically affect acquisition of the traceconditioned EB CR as well as the reversal of EB differentiation (Buchanan and Powell, 1980; Moyer et al., 1990). It has been shown that post-training hippocampal lesions, immediately after training, also dramatically impair later performance of the trace EB CR (Kim et al., 1995). Thus, there is a similarity in the effects of lesions of the mPFC and the hippocampus. Connections between the hippocampus and subicular area to the mPFC are known to exist (Swanson, 1981; Ferino et al., 1987; Chachich et al., 1996). It is thus possible that information processed by the hippocampus and relayed to the $\mathrm{mPFC}$ accounts for these effects of mPFC lesions on trace EB conditioning.

Takehara et al. (2003) as well as Christian and Thompson (2003) suggest that such information results in a reorganization of memory processes from temporary storage in the hippocampus to more permanent storage in the PFC. However, the present results suggest that the $\mathrm{mPFC}$ is important in retrieval and not storage of information, which determines performance of the $\mathrm{EB}$ response. We suggest, based on a wide range of available data (see above), that permanent storage is in the deep nuclei of the cerebellum. Recent neuroanatomical studies have shown connections between the cerebellum and mediodorsal nucleus of the thalamus, which provides the specific thalamic projection neurons to the mPFC. Thus, it is possible that a mPFC-cerebellarthalamic module is necessary for efficient acquisition of the EB CR during trace and reversal conditioning (Powell et al., 2000). Such models have been proposed by other investigators (Houk and Wise, 1995; Weiss and Disterhoft, 1996).

Although the precise mechanisms for these effects are not clearly understood, it is assumed by most that, under ideal conditions (i.e., when the parameters are optimal for acquisition of the response), limbic system input from the hippocampus is not necessary for the memory trace to be carried through the interstimulus interval. However, during trace procedures, in which the CS is not physically present to activate appropriate memory mechanisms, hippocampal input to extrapyramidal structures (which we believe is through the subiculum-mPFC-neostriatal connections) is necessary for the memory trace to persist through the trace period. As noted above, connections also exist between the hippocampus and the $\mathrm{mPFC}$, which suggests that information concerning the status of environmental and somatomotor response selection mechanisms may be routed through the hip- 
pocampus and mPFC to the cerebellum to modify the output of the cerebellur nuclei that determine acquisition of the EB CR under conditions that require limbic and cortical processing (i.e., trace conditioning). In any case, it is apparent that a possible effect of mPFC lesions on the EB CR is related to retrieval as opposed to acquisition and storage mechanisms. Such an analysis could explain the effects of $\mathrm{mPFC}$ lesions on acquisition of the trace versus delay EB CR, in that, from trial to trial and session to session, retrieval of memories associated with execution of the EB $\mathrm{CR}$ are required. $\mathrm{mPFC}$ lesions may thus interfere with such retrieval, which would have a more dramatic effect on trace conditioning. Consequently, the conclusion that retrieval rather than acquisition or storage mechanisms are crucial to mPFC integration of somatomotor classically conditioned responses is not incompatible with the finding that such lesions also affect acquisition of the EB CR using the trace but not the delay paradigm.

\section{References}

Buchanan SL, Powell DA (1980) Divergencies in pavlovian conditioned heart rate and eyeblink responses produced by hippocampectomy in the rabbit (Oyctolagus cuniculus). Behav Neural Biol 30:20-38.

Buchanan SL, Powell DA (1982) Cingulate cortex: its role in pavlovian conditioning. J Comp Physiol Psychol 96:755-774.

Buchanan SL, Valentine JD, Powell DA (1985) Autonomic responses are elicited by electrical stimulation of the medial but not lateral frontal cortex in rabbits. Behav Brain Res 18:51-62.

Buchanan SL, Thompson RH, Maxwell BL, Powell DA (1994) Efferent connections of the medial prefrontal cortex in the rabbit. Exp Brain Res 100:469-483.

Chachich M, Powell DA (1999) Both medial prefrontal and amygdala central nucleus lesions abolish heart rate classical conditioning, but only prefrontal lesions impair reversal of eyeblink differential conditioning. Neurosci Lett 257:151-154.

Chachich M, Penney J, Powell DA (1996) Subicular lesions disrupt but do not abolish classically conditioned bradycardia in rabbits. Behav Neurosci 110:707-717.

Christian KM, Thompson RF (2003) Neural substrates of eyeblink conditioning: acquisition and retention. Learn Mem 11:427-455.

Devinsky O, Morrell MJ, Vogt BA (1995) Contributions of anterior cingulate cortex to behaviour. Brain 118:279-306.

Ferino F, Thierry AM, Glowinski J (1987) Anatomical and electrophysiological evidence for a direct projection from Ammon's horn to the medial prefrontal cortex in the rat. Exp Brain Res 65:421-426.

Frysztak RJ, Neafsey EJ (1991) The effect of medial frontal cortex lesions on respiration, "freezing," and ultrasonic vocalizations during conditioned emotional responses in rats. Cereb Cortex 1:418-425.

Frysztak RJ, Neafsey EJ (1994) The effect of medial frontal cortex lesions on cardiovascular conditioned emotional responses in the rat. Brain Res 643:181-193.

Fuster JM (1997) The prefrontal cortex: anatomy, physiology, and neuropsychology of the frontal lobe. Philadelphia: Lippincott-Raven.
Goldman-Rakic PS (1995) Cellular basis of working memory. Neuron 14:477-485.

Gormezano I (1966) Classical conditioning. In: Experimental methods and instrumentation in psychology (Sidowski JB, ed), pp 385-420. New York: McGraw-Hill.

Houk JC, Wise SP (1995) Distributed modular architectures linking basal ganglia, cerebellum, and cerebral cortex: their role in planning and controlling action. Cereb Cortex 5:95-110.

Kim JJ, Clark RE, Thompson RF (1995) Hippocampectomy impairs the memory of recently, but not remotely, acquired trace eyeblink conditioned responses. Behav Neurosci 109:195-203.

Kimble GA (1961) Hilgard and Marquis' conditioning and learning. New York: Appleton-Century-Crofts.

Kronforst-Collins MA, Disterhoft JF (1998) Lesions of the caudal area of rabbit medial prefrontal cortex impair trace eyeblink conditioning. Neurobiol Learn Mem 69:147-162.

McLaughlin J, Skaggs H, Churchwell J, Powell DA (2002) Medial prefrontal cortex and pavlovian conditioning: trace versus delay conditioning. Behav Neurosci 116:37-47.

Moyer Jr JR, Deyo RA, Disterhoft JF (1990) Hippocampectomy disrupts trace eye-blink conditioning in rabbits. Behav Neurosci 104:243-252.

Powell DA, Watson K, Maxwell B (1994) Involvement of subdivisions of the medial prefrontal cortex in learned cardiac adjustments. Behav Neurosci 108:294-307.

Powell DA, McLaughlin J, Chachich M (2000) Classical conditioning of autonomic and somatomotor responses and their central nervous system substrates. In: Eyeblink classical conditioning, Vol 2, Animal models (Steinmetz JE, Woodruff-Pak DS, eds), pp 257-286. Boston: Kluwer.

Powell DA, Skaggs H, Churchwell J, McLaughlin J (2001) Post-training lesions of the medial prefrontal cortex impair performance of pavlovian eyeblink conditioning but have no effect on concomitant heart rate changes. Behav Neurosci 115:1029-1038.

Powell DA, Churchwell J, Burriss L (2005) Medial prefrontal lesions and pavlovian eyeblink and heart rate conditioning: effects of partial reinforcement on delay and trace conditioning in rabbits (Oryctolagus cuniculus). Behav Neurosci 119:180-189.

Shek JW, Wen GY, Wisniewski HM (1986) Atlas of the rabbit brain and spinal cord. New York: Karger.

Swanson LW (1981) A direct projection from Ammon's horn to prefrontal cortex in the rat. Brain Res 217:150-154.

Takehara K, Kawahara S, Kirino Y (2003) Time-dependent reorganization of the brain components underlying memory retention in trace eyeblink conditioning. J Neurosci 23:9897-9905.

Thompson RF, Steinmetz JE, Woodruff-Pak DS (2000) Discovering the brain substrates of eyeblink classical conditioning. In: Eyeblink classical conditioning, Vol 2, Animal models (Steinmetz JE, Woodruff-Pak DS, eds), pp. 17-49. Boston: Kluwer.

Weible AP, McEchron MD, Disterhoft JF (2000) Cortical involvement in acquisition and extinction of trace eyeblink conditioning. Behav Neurosci 114:1058-1067.

Weiss C, Disterhoft JF (1996) Eyeblink conditioning, motor control, and the analysis of limbic-cerebellar interactions. Behav Brain Sci 19:479-527. 